\title{
Evaluation of a novel noninvasive ICP monitoring device in patients undergoing invasive ICP monitoring: preliminary results
}

\author{
Oliver Ganslandt, MD, ${ }^{1}$ Stylianos Mourtzoukos, MD, ${ }^{1}$ Andreas Stadlbauer, PhD, ${ }^{2}$ \\ Björn Sommer, MD, ${ }^{2}$ and Rudolf Rammensee, MD$^{2}$ \\ ${ }^{1}$ Department of Neurosurgery, Klinikum Stuttgart; and 2Department of Neurosurgery, University of Erlangen, Germany
}

\begin{abstract}
OBJECTIVE There is no established method of noninvasive intracranial pressure (NI-ICP) monitoring that can serve as an alternative to the gold standards of invasive monitoring with external ventricular drainage or intraparenchymal monitoring. In this study a new method of $\mathrm{NI}-\mathrm{ICP}$ monitoring performed using algorithms to determine ICP based on acoustic properties of the brain was applied in patients undergoing invasive ICP (I-ICP) monitoring, and the results were analyzed.

METHODS In patients with traumatic brain injury and subarachnoid hemorrhage who were undergoing treatment in a neurocritical intensive care unit, the authors recorded ICP using the gold standard method of invasive external ventricular drainage or intraparenchymal monitoring. In addition, the authors simultaneously measured the ICP noninvasively with a device (the HS-1000) that uses advanced signal analysis algorithms for acoustic signals propagating through the cranium. To assess the accuracy of the NI-ICP method, data obtained using both I-ICP and NI-ICP monitoring methods were analyzed with MATLAB to determine the statistical significance of the differences between the ICP measurements obtained using $\mathrm{NI}-\mathrm{ICP}$ and I-ICP monitoring.
\end{abstract}

RESULTS Data were collected in 14 patients, yielding 2543 data points of continuous parallel ICP values in recordings obtained from I-ICP and NI-ICP. Each of the 2 methods yielded the same number of data points. For measurements at the $\geq 17-\mathrm{mm} \mathrm{Hg}$ cutoff, which was arbitrarily chosen for this preliminary analysis, the sensitivity and specificity for the $\mathrm{NI}-\mathrm{ICP}$ monitoring were found to be 0.7541 and 0.8887 , respectively. Linear regression analysis indicated that there was a strong positive relationship between the measurements. Differential pressure between $\mathrm{NI}-\mathrm{ICP}$ and I-ICP was within \pm $3 \mathrm{~mm} \mathrm{Hg}$ in $63 \%$ of data-paired readings and within $\pm 5 \mathrm{~mm} \mathrm{Hg}$ in $85 \%$ of data-paired readings. The receiver operating characteristic-area under the curve analysis revealed that the area under the curve was 0.895 , corresponding to the overall performance of $\mathrm{NI}-\mathrm{ICP}$ monitoring in comparison with I-ICP monitoring.

CONCLUSIONS This study provides the first clinical data on the accuracy of the HS-1000 NI-ICP monitor, which uses advanced signal analysis algorithms to evaluate properties of acoustic signals traveling through the brain in patients undergoing I-ICP monitoring. The findings of this study highlight the capability of this $\mathrm{NI}-\mathrm{ICP}$ device to accurately measure ICP noninvasively. Further studies should focus on clinical validation for elevated ICP values.

https://thejns.org/doi/abs/10.3171/2016.11.JNS152268

KEY WORDS intracranial pressure monitoring; diagnostic technique; traumatic brain injury

$\mathrm{R}$ ECORDING and interpretation of intracranial pressure (ICP) is one of the most important diagnostic tools in neurosurgery and neurology. ${ }^{9}$ There is growing evidence that ICP monitoring and protocol-driven therapy help to improve clinical outcome in a number of pa- thologies such as traumatic brain injury (TBI), brain infections such as meningitis, and brain tumors. ${ }^{22}$ To date, ICP monitoring is still an invasive procedure in which a burr hole is used to introduce a transducer-coupled probe into the brain parenchyma or into the ventricles. Invasive ICP

ABBREVIATIONS AUC = area under the curve; $\mathrm{CPP}=$ cerebral perfusion pressure; EVD = external ventricular drainage; $\mathrm{I}-\mathrm{ICP}=$ invasive intracranial pressure; ICU = intensive care unit; IPM = intraparenchymal monitor; NI-ICP = noninvasive ICP; ONSD = optic nerve sheath diameter; ROC = receiver operating characteristic; TBI = traumatic brain injury.

SUBMITTED September 27, 2015. ACCEPTED November 17, 2016.

INCLUDE WHEN CITING Published online August 8, 2017; DOI: 10.3171/2016.11.JNS152268. 
monitoring coupled with the use of external ventricular drainage (EVD) is still considered the gold standard of ICP monitoring and is part of the standard patient management in the intensive care unit (ICU). ${ }^{3,8,8,18,20,31,34}$ The invasive nature of this method and the methodology itself makes invasive ICP (I-ICP) monitoring both a potentially harmful and technically difficult technique. ${ }^{17,23}$ The main concerns with using EVD include an associated high infection rate, which ranges from $3.4 \%$ to $32.2 \% ;^{7}$ the rate of malplacement of the ventricular catheter, which in one study was as high as $12.3 \%: 28$ and the problem of technically inaccurate recordings due to calibration errors. ${ }^{24}$

When considering the disadvantages of I-ICP monitoring, it is understandable that efforts have been made to introduce an alternative measure that would allow for noninvasive ICP (NI-ICP) monitoring performed using surrogate parameters that correspond to the ICP but can be recorded from the outside of the skull. Of these techniques, transcranial Doppler ultrasonography, ${ }^{32}$ tympanic membrane displacement, ${ }^{12}$ and optic nerve sheath diameter (ONSD) $)^{19}$ have been investigated. However, all methods have distinctive limitations that have prevented them from being used as replacements for invasive monitoring. Raboel et al. stated that NI-ICP monitoring techniques are inaccurate and cannot be used as an alternative to invasive techniques. ${ }^{24}$

In this study we investigated a new approach to NI-ICP monitoring. The NI-ICP monitor used in our study consists of a device (HS-1000; HeadSense Medical, Ltd.) that generates an acoustic signal that propagates through the cranium. The modulations of the signal in different physiological and pathophysiological conditions are processed using advanced signal analysis. The device displays the recorded ICP as quantitative data (in $\mathrm{mm} \mathrm{Hg}$ ) but is capable of analyzing a multitude of other parameters through evaluation of the respiratory and cardiac cycles and intracranial vessel properties, along with other parameters. In this study we focused on a correlative investigation in which ICP measurements obtained using the NI-ICP monitor were compared with those obtained using invasive EVD and intraparenchymal monitoring techniques.

\section{Methods}

Patients and/or next of kin consented to participation in this study. In most cases consent was given by next of kin and was later affirmed by the patient if possible. This was in congruence with the local ethics committee requirements. The study was approved by the local ethics committees of our institutions. The study design was a prospective parallel comparison of I-ICP and NI-ICP values. The I-ICP measurements were obtained with one of 2 methods, the EVD I-ICP monitor (Spiegelberg GmbH \& Co. KG) or the intraparenchymal ICP monitor (IPM) (Codman ICP MicroSensor; Codman, Johnson \& Johnson), and data from the 2 methods were combined. The NI-ICP values were obtained with the HS-1000 (HeadSense Medical, Ltd.).

The study was performed at the Departments of Neurosurgery at the University Hospital of Erlangen and Klinikum Stuttgart. Fourteen patients were enrolled, all of whom were undergoing continuous I-ICP monitoring in a neurocritical ICU because of either TBI or subarachnoid hemorrhage. Data were collected and stored for further retrospective analysis of the pseudonymized digital recordings. Some of the patients were treated with EVD; continuous I-ICP monitoring was performed in conjunction with the EVD system as part of the standard ICU management. We also included patients in whom an IPM was used for I-ICP monitoring. All patients underwent NIICP monitoring. Exclusion criteria included ear disease or ear trauma, rhinorrhea or otorrhea, skull defect, pregnancy, and severe open head trauma, as well as age younger than 18 years. Throughout all parallel ICP monitoring sessions, each patient was positioned supine with the head placed at a $30^{\circ}$ angle.

\section{Invasive ICP Monitoring}

The intraparenchymal monitoring involved intraparenchymal placement of a catheter. The pressure value, which was measured in $\mathrm{mm} \mathrm{Hg}$, was relayed electronically via a strain-gauge microchip located at the tip of the catheter, which was mounted in a titanium case at the tip of a $100-\mathrm{cm}$ flexible nylon tube. ${ }^{16}$ Ventricular ICP monitoring (performed with EVD) is considered a gold standard for ICP monitoring and has been described previously. ${ }^{30}$ The EVD system is based on a probe, which is inserted via Kocher's point into the lateral ventricle and is used for both drainage of CSF and pressure monitoring. The EVD system diverts CSF by using a combination of gravity and intracerebral pressure. The drainage rate depends on the height at which the EVD system is placed relative to the patient's anatomy. When the EVD valve is closed, the ICP measurement is attainable. Continuous ICP monitoring cannot be performed during CSF drainage; when the EVD valve is open, the ICP value is not accurate and no measurements should be taken. In the present study, if the ventricular catheter was open for drainage, the line was closed and the system was allowed to equilibrate for 2 minutes before a reading was taken.

\section{Noninvasive ICP Monitoring}

For NI-ICP monitoring we used the HS-1000 device (HeadSense Medical Ltd.). This device uses advanced signal analysis algorithms that evaluate an acoustic signal. This signal includes a short beep at $66 \mathrm{~dB}$ for approximately 6 seconds, which is emitted from the ipsilateral ear. It then propagates through the cranium and is detected together with other physiological sounds from the brain by the receiving sensor located in the contralateral ear. The HS-1000 data are continuously recorded in 6-second epochs on the corresponding device's monitor. At the end of signal transmission, the HS-1000 device's monitor is programmed to automatically take a snapshot of the IICP monitor screen or the patient's monitor (including date and time, ICP value in $\mathrm{mm} \mathrm{Hg}$, and blood pressure). These values are saved in a database for future comparative evaluations. Each NI-ICP monitoring session was approximately $30-60$ minutes long, as the patient's clinical condition permitted. The limiting factor for the length of the monitoring session was the patient's ability to tolerate closure of the EVD valve throughout the monitoring 
session. In cases in which the patient's condition did not allow continuous recording (e.g., when the patient's CSF had to be drained or the patient's management required some form of intervention), the recording was stopped. It was resumed only when the patient's state allowed proper recording conditions (lying in a supine position without any movement or intervention from the clinicians). Once all recordings were complete, the ICP values from the IICP monitor were saved in the study's database. For purposes of analysis, ranges of I-ICP were formulated and the data pairs of I-ICP and NI-ICP were randomly sampled to accrue the number of values needed for analysis and to calculate sensitivity and specificity (Fig. 1).

\section{Parallel ICP Monitoring}

Each parallel monitoring interval in which both the NIICP and I-ICP methods were used consisted of between 30 minutes and 6 hours of aggregate recording performed either continuously or in separate recording sessions as the patient's condition allowed, which was repeated 3 times per day for up to 48 hours. There were a minimum of 3 to as many as 8640 data points per patient. Both EVD and intraparenchymal monitoring were assumed to be gold standards; we recorded in parallel from both of the I-ICP monitors and the NI-ICP monitor and compared the values. The raw data on acoustic signals collected by the HS1000 (generated signal and accompanying physiological signals) were analyzed for approximately 5 seconds, and the NI-ICP value was calculated using a proprietary signal-processing algorithm and displayed to the user in $\mathrm{mm}$ $\mathrm{Hg}$. Each NI-ICP monitoring cycle lasted approximately 15 seconds (including 6 seconds for the signal to be transmitted and received, approximately 5 seconds for snapshots to be taken, and 4 seconds for calculation of the ICP value). This translates to approximately 4 measurements per minute. We defined the "mean ICP" independent score as the corresponding I-ICP value that is determined every consecutive period of approximately 11 seconds as the sum of sample values divided by the number of samples. This is an acceptable time window compared with current state-of-the-art methodologies that range between 5 and 15 seconds in duration. During the test, no manipulations to change the ICP were done, and nothing was changed in the usual patient management protocols.

\section{Statistical Analysis}

Data were analyzed using MATLAB. Pearson correlation analysis of the I-ICP and NI-ICP values was completed to compare the ICP values from all devices. For 95\% confidence, a $\mathrm{p}$ value $\leq 0.05$ was considered statistically significant. The difference between the NI-ICP and I-ICP monitoring results was plotted against the mean of the 2 measures (I-ICP and NI-ICP). Receiver operating characteristics (ROCs) with the area under the curve (AUC) were calculated to present the tradeoff between sensitivity and specificity for all measurements at the $\geq 15 \mathrm{~mm} \mathrm{Hg}$ cutoff. The AUC was calculated for all data points to provide an estimator for the cumulative measurements. Differential pressure (in $\mathrm{mm} \mathrm{Hg}$ ) was defined as the difference between I-ICP and NI-ICP per data point. The Bland-Altman

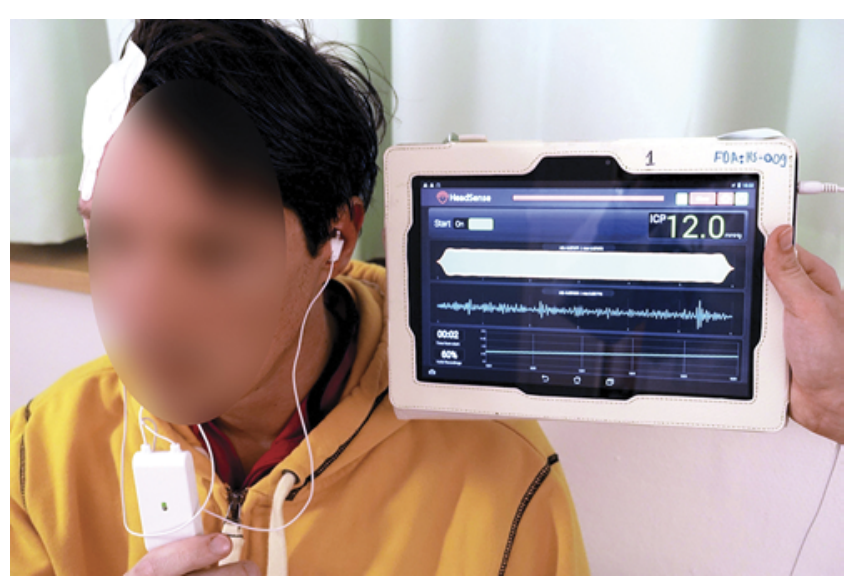

FIG. 1. Photograph showing the HS-1000 device during a recording session. Figure is available in color online only.

method was used to examine the percentages of total data points below the $6-\mathrm{mm} \mathrm{Hg}$ and $5-\mathrm{mm} \mathrm{Hg}$ differential pressure. The relationship of the paired data points was assessed using linear regression analysis, which showed the relationship between NI-ICP measurements and those obtained using the I-ICP. The slope of the line of best fit was used to examine the predictability of I-ICP from NIICP measurements.

\section{Results}

\section{Parallel ICP Monitoring}

Fourteen patients met the study's inclusion criteria (Table 1); 1 patient was excluded because of a skull defect. The data from each patient were collected over a session of approximately 30 minutes to 1 hour. Throughout the study, no adverse effects were observed. ICP data were collected from all enrolled patients, yielding 2543 data points of continuous parallel ICP values in recordings obtained using I-ICP and NI-ICP devices. The I-ICP results reported are a combination of data obtained with the EVD (12 patients) and IPM (2 patients) I-ICP methods. Differences between I-ICP and NI-ICP readings (i.e., differential pressure of direct readings) were obtained. Differential pressures within $\pm 3 \mathrm{~mm} \mathrm{Hg}$ were observed in 1606 (63\%) readings, and differential pressures within $\pm 5 \mathrm{~mm}$ $\mathrm{Hg}$ were observed in 2154 (85\%) readings. The numbers of measurements for each patient varied, but the mean ICP values were $10 \pm 6.1 \mathrm{~mm} \mathrm{Hg}$ (with a range of $0-26 \mathrm{~mm}$ $\mathrm{Hg}$ ) and $9.5 \pm 4.7 \mathrm{~mm} \mathrm{Hg}$ (with a range of $0-21 \mathrm{~mm} \mathrm{Hg}$ ) for the I-ICP and NI-ICP measures, respectively.

The ICP values from an I-ICP session were plotted against the NI-ICP values (Fig. 2). The trend plot revealed a Pearson $r$ correlation of 0.82 between the measurements and a 95\% CI for $\mathrm{r}$ of $0.81-0.83(\mathrm{p}<0.0001)$.

A Bland-Altman plot, in which the difference between ICP readings obtained using the I-ICP and NI-ICP were plotted against the ICP as the average of the 2 methods, showed an average bias of $0.54 \mathrm{~mm} \mathrm{Hg}$, with $95 \%$ limits of agreement of $-6.3 \mathrm{~mm} \mathrm{Hg}$ and $7.38 \mathrm{~mm} \mathrm{Hg}$ (Fig. 3).

Linear regression analysis yields a line of best fit with the following equation: $y=0.6357 * x+3.123$ (Fig. 4). This 
TABLE 1. Baseline characteristics of 14 patients enrolled in the ICP monitoring study

\begin{tabular}{lc}
\hline \multicolumn{1}{c}{ Variable } & No. of Patients $(\%)^{*}$ \\
\hline Sex & $11(79)$ \\
\hline Male & $4(29)$ \\
\hline Female & $2(14)$ \\
\hline Age range in yrs & $3(21)$ \\
\hline $40-49$ & $6(43)$ \\
\hline $50-59$ & $4(29)$ \\
\hline $60-69$ & $6(43)$ \\
\hline $70-79$ & $1(7)$ \\
\hline Diagnosis & $6(43)$ \\
\hline Subarachnoid hemorrhage & $1(7)$ \\
\hline Acute subdural hematoma & \\
\hline Intracranial hemorrhage & \\
\hline Malignant MCA infarction & \\
\hline MCA = middle cerebral artery. \\
* The totals for sex and age add up to 15, but 1 patient was excluded from \\
monitoring because of a skull defect.
\end{tabular}

equation best predicts the NI-ICP results from the I-ICP results. It also shows that there is a strong positive relationship between the measurements obtained with the NI-ICP and I-ICP devices.

As presented in Fig. 5, ROC curve analysis revealed an AUC of 0.895, corresponding to overall performance of the NI-ICP to correctly identify I-ICP values. For measurements at the $\geq 17-\mathrm{mm} \mathrm{Hg}$ cutoff, which was arbitrarily chosen for this preliminary analysis, the sensitivity and specificity for the NI-ICP monitoring were found to be 0.7541 and 0.8887 , respectively.

\section{Discussion}

This study provides the first clinical data on the accuracy of the HS-1000 device (an NI-ICP monitor) in patients undergoing an I-ICP monitoring procedure for various clinical conditions resulting from TBI and/or SAH. The data points of continuous parallel recordings of ICP values were obtained from I-ICP and NI-ICP simultaneously. The overall findings showed that there was a good correlation between the measurements obtained with these 2 modalities $(r=0.82 ; p<0.0001)$, revealing the potential of the noninvasive HS-1000 monitor for measurement of quantitative ICP. The average ICP readings for the I-ICP and NIICP methods were $10 \pm 6.1 \mathrm{~mm} \mathrm{Hg}$ and $9.5 \pm 4.7 \mathrm{~mm} \mathrm{Hg}$, respectively. For measurements at the $\geq 17-\mathrm{mm} \mathrm{Hg}$ cutoff, sensitivity and specificity for the NI-ICP monitoring were 0.7541 and 0.8887 , respectively. Bias and agreement analysis performed using the Bland-Altman plot showed good concordance between the 2 measures. We have demonstrated a systematic way to incorporate NI-ICP monitoring via use of the HS-1000 device in a neurocritical ICU environment.

Currently, I-ICP monitoring is a fundamental tool that provides primary surveillance management of patients in neurocritical ICUs. For the I-ICP measurement modality, probes are inserted into the brain parenchyma or ven-

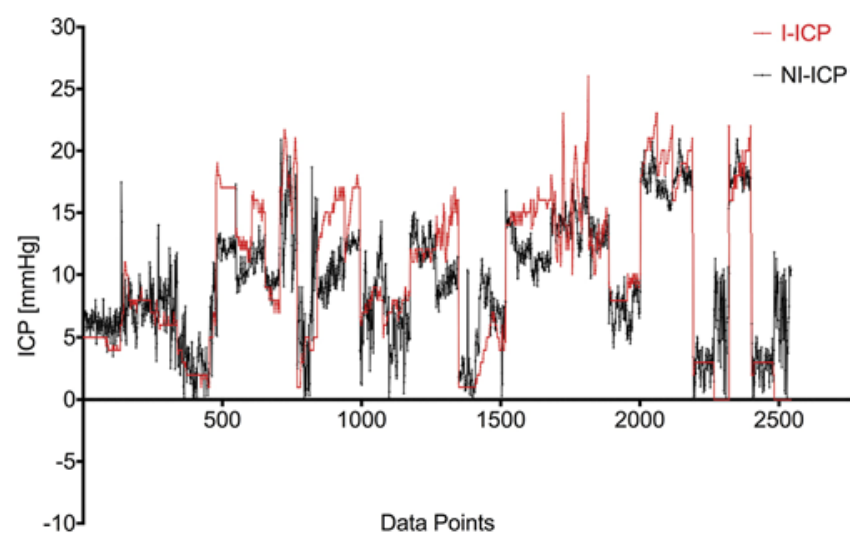

FIG. 2. Trend plot showing 2348 continuous parallel recordings of ICP values obtained from I-ICP compared with NI-ICP. Pearson $r=0.824$, and $95 \% \mathrm{Cl}$ for $\mathrm{r}: 0.81-0.83(\mathrm{p}<0.0001)$. Figure is available in color online only.

tricles. The predominant methods of invasive continuous ICP monitoring are performed via intraparenchymal or intraventricular probes. Arguably, the latter is considered the gold standard for ICP monitoring. The EVD system allows therapeutic CSF drainage and clearance of intraventricular blood..$^{2,14,27}$ More importantly, an elevated ICP is considered a medical emergency and can be managed medically via placement of an EVD system. The fundamental indication for management of elevated ICP is to prevent secondary ischemia due to the decrease in cerebral perfusion, which may lead to brain herniation. ${ }^{3}$ The methodology of ICP monitoring via an EVD system is meant to allow continuous monitoring as well as drainage, but not to perform both simultaneously. The standard of care is to drain by opening the EVD valve (transducer) only for elevations at or above the $20 \mathrm{~mm} \mathrm{Hg}$ threshold (ICP $\geq 20 \mathrm{~mm} \mathrm{Hg}$ ) and to relieve elevated ICP. In clinical practice, the EVD valve is kept closed for ICP measurements and to assess the continued need for CSF drainage. The inability of IPMs to allow drainage does not impede their usefulness in ICP monitoring, yet the accuracy level may be compromised when subjected to varying degrees of zero drift during the monitoring, due to the inherent inability to recalibrate the sensor in situ. ${ }^{4,15}$

As for the indications, the ICP threshold at which herniation may occur is not absolute, and may vary between patients in regard to interindividual heterogeneity and the location of the intracranial mass lesion. ${ }^{1}$ Cerebral perfusion can be monitored indirectly by measuring cerebral perfusion pressure (CPP), which is equal to the difference between mean arterial pressure and ICP. The CPP can be evaluated and managed by manipulation of arterial pressure, and is derived from ICP-monitored patients. ${ }^{15}$

The above-mentioned threshold of $20 \mathrm{~mm} \mathrm{Hg}$ is not a clear cutoff, yet it is considered a clinical target for ICP management for the treatment of severe TBI. Evidently, research supports ICP thresholds of 20-25 $\mathrm{mm} \mathrm{Hg}$ to promote investigation and treatment of elevated ICP. ${ }^{5}$ Nevertheless, in a meta-analysis investigating ICP monitoring in patients with TBI, the authors concluded that it is still essential to identify the optimal threshold value of ICPdirected treatment, even in recent reviews of ICP monitor- 


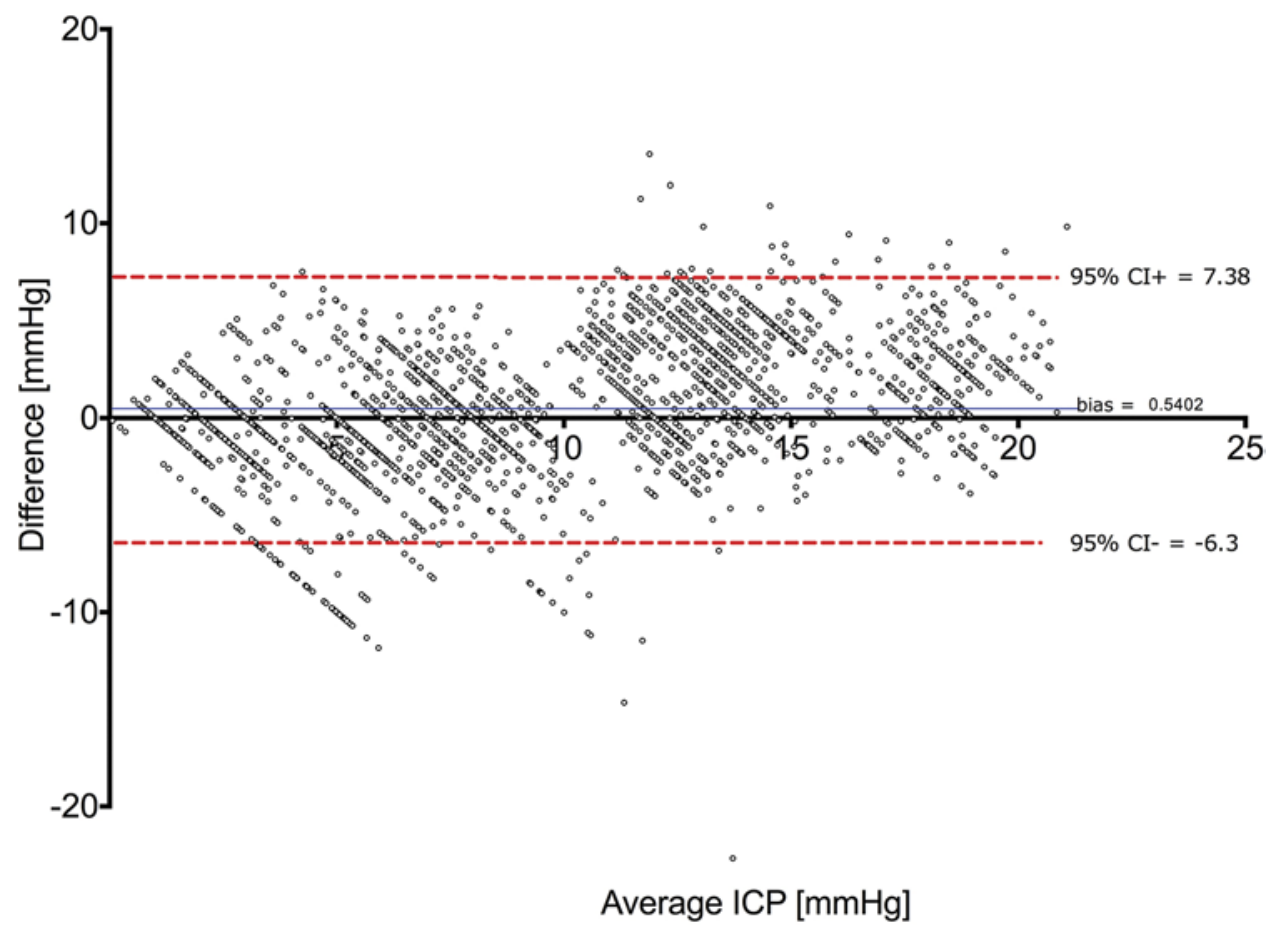

FIG. 3. Bland-Altman plot showing a good concordance between I-ICP and NI-ICP values. Figure is available in color online only.

ing. ${ }^{33}$ We introduced a new method for continuous NI-ICP monitoring and compared it to the main state-of-the-art methods currently available in neurocritical ICUs.

Although, for reasons described below, we consider our NI-ICP modality to be highly accurate, our comparison was confined to protocol-driven management for elevated
ICP, and therefore we were unable to compare data points when the patient's condition did not allow continuous recording. During this study, data collection was possible only when the EVD valve was closed, unless the patient's management protocol required some form of intervention (based on a diagnosis of elevated ICP). Therefore, we were

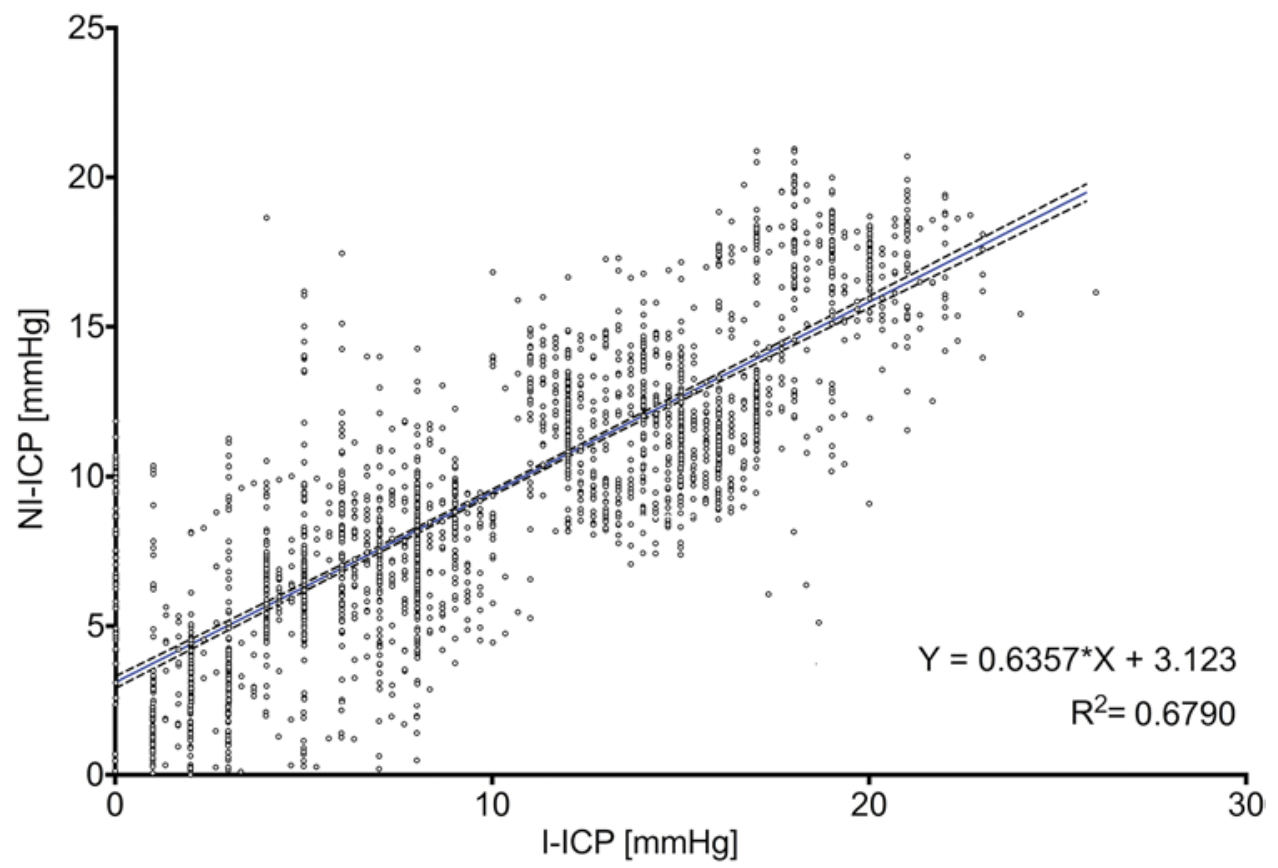

FIG. 4. Linear regression analysis showing a strong positive relationship between the NI-ICP and I-ICP values. Figure is available in color online only. 


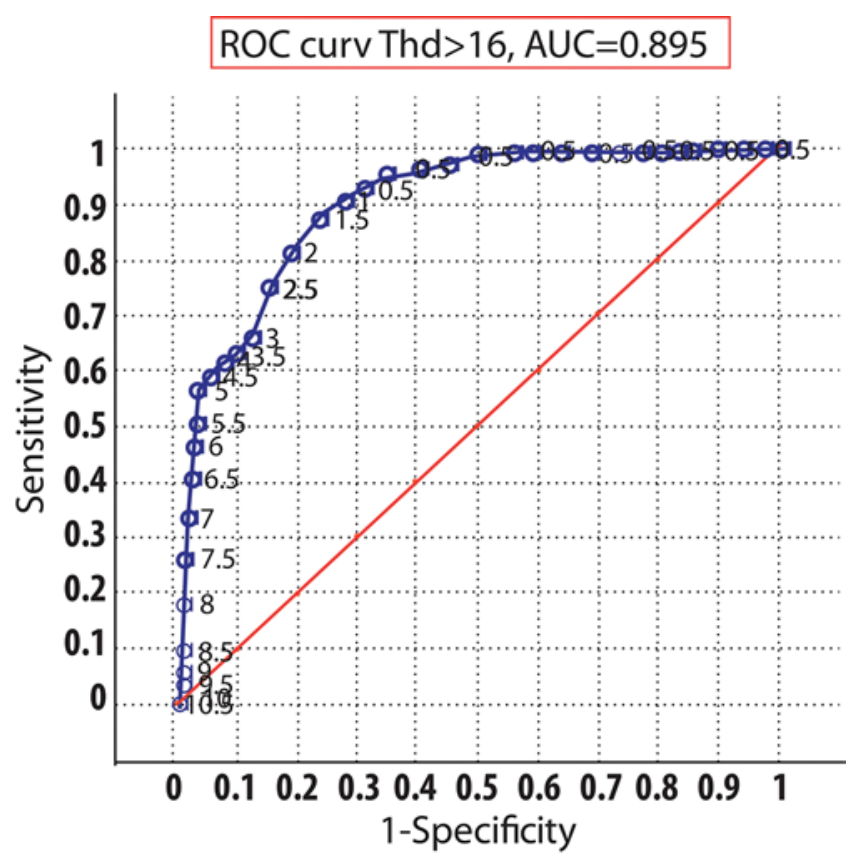

FIG. 5. The ROC curve for all measurements. The AUC was 0.895. Thd $=$ threshold. Figure is available in color online only.

able to obtain measurements within a range of $0-26 \mathrm{~mm}$ $\mathrm{Hg}$ (mean ICP $10 \pm 6.1 \mathrm{~mm} \mathrm{Hg}$ ) or $0-21 \mathrm{~mm} \mathrm{Hg}$ (mean ICP $9.5 \pm 4.7 \mathrm{~mm} \mathrm{Hg}$ ) for the I-ICP and NI-ICP measures, respectively.

Continuous ICP monitoring is of paramount importance for the treatment of elevated ICP; it is essential for maintaining a satisfactory CPP and to avoid inadequate oxygen delivery that may lead to secondary injury. Although ICP measurement is widely accepted as a core parameter in most neurocritical ICUs worldwide, invasive modalities have a number of drawbacks. The main challenge is that both intraparenchymal and intraventricular catheters have risks of complications; i.e., infection or postoperative hemorrhage. Moreover, invasive monitoring requires constant management and continuous monitoring, which may be burdensome to the ICU staff and in some cases may limit ICP monitoring. ${ }^{21}$ The EVD system presents some clear advantages over intraparenchymal monitoring because it allows CSF drainage, which may significantly decrease the need for surgical decompression..$^{15}$ However, it is associated with a higher risk of infection compared with intraparenchymal monitoring. ${ }^{26}$ As mentioned above, drainage requires opening the EVD valve (i.e., the mounted transducer) and exposing it to atmospheric pressure. Furthermore, drainage requires completing a zeroing procedure to obtain the zero reference point, which hinders the integrity of the sterile closed drainage system. This limits the accuracy of ICP reading to periods in which it is clinically possible to close the mounted transducer. ${ }^{29}$ The accuracy of ICP readings via the EVD system is also dependent on maintaining the patient in a constant position where the transducer is fixed at the level of the foramen of Monro. This is done by creating an imaginary point of intersection between the ipsilateral medial canthus of the orbit and the tragus of the ear. ${ }^{14}$ All of these considerations present significant drawbacks and limit the accuracy of the EVD system's ICP monitors. However, EVD devices are considered the most accurate, reliable, and cost-effective ICP monitoring methodology today, ${ }^{2,15}$ with functional accuracy of $87 \% .^{14}$

To overcome these obstacles, a variety of NI-ICP monitoring modalities have been explored in recent years, but according to several comparative analyses, none are as accurate as the invasive modalities. ${ }^{24,27}$ Yet, as the results of these analyses indicate, some noninvasive modalities may yield more clinically useful results in the presence of elevated ICP: these include ultrasound and color Doppler ultrasonography. Ultrasound measures of the ONSD present a unique noninvasive tool for detecting ICP hypertension. Research suggests ONSD as a possible indicator of elevated ICP. The underlying principle states that an increase in ICP results in distension of the ONSD (millimetric increase) within seconds. The accepted consensus for an elevated ICP is ONSD above a threshold of $0.5 \mathrm{~cm} .{ }^{11,25}$ Rajajee et al. $^{25}$ conducted a comparison between ONSD and I-ICP measurements, EVD, and intraparenchymal monitoring, indicating that the optimal ONSD for detection of ICP $\geq$ $20 \mathrm{~mm} \mathrm{Hg}$ was $\geq 0.48 \mathrm{~cm}$ (sensitivity $96 \%$ and specificity 94\%). Spearman's correlation coefficient of an ONSD of $0.52 \mathrm{~cm}$ for detection of ICP $\geq 20 \mathrm{~mm} \mathrm{Hg}$ was $0.73 .{ }^{25}$ The main limitation of this method is that the measurement accuracy corresponds to the operator's experience and equipment, as well as the fact that the ONSD criterion (i.e., cutoff for ICP hypertension) is not well established..$^{13,25}$ All of the aforementioned drawbacks can be counterproductive to the implementation of this method and its effectiveness in ICUs.

In this study, differences between data-paired readings were also calculated for each patient recording. Major differences in the mean ICP were first observed by Fernandes et al., who demonstrated sudden shifts in the mean ICP while simultaneously using 2 separate IPMs (1 Codman microsensor and 1 Camino fiberoptic device).$^{10}$ In our study, the differential pressure between NI-ICP and I-ICP was within $\pm 3 \mathrm{~mm} \mathrm{Hg}$ in $63 \%$ of data-paired readings and within $\pm 5 \mathrm{~mm} \mathrm{Hg}$ in $85 \%$ of data-paired readings. We believe these findings to be particularly interesting because similar differences have been reported for studies comparing 2 invasive modalities, intraventricular and intraparenchymal ICP. Chambers et al. reported similar results (79\%) that were within $\pm 5 \mathrm{~mm} \mathrm{Hg}$ for simultaneously comparisons of readings from the Spiegelberg transducer and the EVD system. ${ }^{6}$ With the HS-1000, the mean difference between ICP readings obtained using the I-ICP and NI-ICP is plotted against the ICP as the average of the 2 methods, and shows an average bias of $0.54 \mathrm{~mm} \mathrm{Hg}$, with $95 \%$ limits of agreement of $-6.3 \mathrm{~mm} \mathrm{Hg}$ and $7.38 \mathrm{~mm} \mathrm{Hg}$. Similarly, reported comparisons between the EVD system and intraparenchymal monitoring measurements obtained using different modalities were consistent with the results of the mean ICP differences in our study. Lescot et al. found a bias of $-0.6 \mathrm{~mm} \mathrm{Hg}$ with $95 \%$ limits of agreement of -8.1 $\mathrm{mm} \mathrm{Hg}$ and $6.9 \mathrm{~mm} \mathrm{Hg}$ between the Pressio IPM and the EVD system, and $0.3 \mathrm{~mm} \mathrm{Hg}$ and limits of agreement of -6.6 and $7.1 \mathrm{~mm} \mathrm{Hg}$ between the Codman IPM and the EVD system. ${ }^{17}$ 


\section{Conclusions}

Further investigation of the HS-1000 ICP monitor should focus on the accuracy of elevated ICP values. The findings of this study highlight the capability of the HS1000 to provide information about normal and elevated ICP levels in a clinical setting. The HS-1000 could be extremely beneficial as an adjunct modality by assisting clinicians in deciding if a patient requires an I-ICP monitor. It may also serve as a unique tool for continuous ICP monitoring, especially in third-world ICUs, in which the sanitation level and general care are not comparable to those in first-world, Western hospitals. The use of this NIICP monitoring device may help avoid the immediate risk of infection and/or hemorrhage associated with the use of I-ICP methods and reduce the morbidity and mortality rates in neurocritical ICUs.

\section{References}

1. Andrews BT, Chiles BW III, Olsen WL, Pitts LH: The effect of intracerebral hematoma location on the risk of brain-stem compression and on clinical outcome. J Neurosurg 69:518522, 1988

2. Berlin T, Murray-Krezan C, Yonas H: Comparison of parenchymal and ventricular intracranial pressure readings utilizing a novel multi-parameter intracranial access system. Springerplus 4:10, 2015

3. Bhatia A, Gupta AK: Neuromonitoring in the intensive care unit. I. Intracranial pressure and cerebral blood flow monitoring. Intensive Care Med 33:1263-1271, 2007

4. Bratton SL, Chestnut RM, Ghajar J, McConnell Hammond FF, Harris OA, Hartl R, et al: Guidelines for the management of severe traumatic brain injury. VII. Intracranial pressure monitoring technology. J Neurotrauma 24 (Suppl 1):S45S54, 2007 (Erratum in J Neurotrauma 25:276-278, 2008)

5. Bratton SL, Chestnut RM, Ghajar J, McConnell Hammond FF, Harris OA, Hartl R, et al: Guidelines for the management of severe traumatic brain injury. VIII. Intracranial pressure thresholds. J Neurotrauma 24 (Suppl 1):S55-S58, 2007 (Erratum in J Neurotrauma 25:276-278, 2008)

6. Chambers IR, Siddique MS, Banister K, Mendelow AD: Clinical comparison of the Spiegelberg parenchymal transducer and ventricular fluid pressure. J Neurol Neurosurg Psychiatry 71:383-385, 2001

7. Collins CD, Hartley JC, Chakraborty A, Thompson DN: Long subcutaneous tunnelling reduces infection rates in paediatric external ventricular drains. Childs Nerv Syst 30:1671-1678, 2014

8. Cremer OL: Does ICP monitoring make a difference in neurocritical care? Eur J Anaesthesiol Suppl 42:87-93, 2008

9. Czosnyka M, Pickard JD: Monitoring and interpretation of intracranial pressure. J Neurol Neurosurg Psychiatry 75:813-821, 2004

10. Fernandes HM, Bingham K, Chambers IR, Mendelow AD: Clinical evaluation of the Codman microsensor intracranial pressure monitoring system. Acta Neurochir Suppl 71:4446, 1998

11. Galetta S, Byrne SF, Smith JL: Echographic correlation of optic nerve sheath size and cerebrospinal fluid pressure. J Clin Neuroophthalmol 9:79-82, 1989

12. Gwer S, Sheward V, Birch A, Marchbanks R, Idro R, Newton $\mathrm{CR}$, et al: The tympanic membrane displacement analyser for monitoring intracranial pressure in children. Childs Nerv Syst 29:927-933, 2013

13. Hightower S, Chin EJ, Heiner JD: Detection of increased intracranial pressure by ultrasound. J Spec Oper Med 12:19-22, 2012

14. Kakarla UK, Kim LJ, Chang SW, Theodore N, Spetzler RF: Safety and accuracy of bedside external ventricular drain placement. Neurosurgery 63 (1 Suppl 1):ONS162-ONS167, 2008

15. Kasotakis G, Michailidou M, Bramos A, Chang Y, Velmahos $\mathrm{G}$, Alam H, et al: Intraparenchymal vs extracranial ventricular drain intracranial pressure monitors in traumatic brain injury: less is more? J Am Coll Surg 214:950-957, 2012

16. Koskinen LO, Olivecrona M: Intracranial pressure monitoring using the Codman MicroSensor. Neurosurgery 67:221, 2010 (Letter)

17. Lescot T, Reina V, Le Manach Y, Boroli F, Chauvet D, Boch AL, et al: In vivo accuracy of two intraparenchymal intracranial pressure monitors. Intensive Care Med 37:875879,2011

18. Lozier AP, Sciacca RR, Romagnoli MF, Connolly ES Jr: Ventriculostomy-related infections: a critical review of the literature. Neurosurgery 51:170-182, 2002

19. Maissan IM, Dirven PJ, Haitsma IK, Hoeks SE, Gommers D, Stolker RJ: Ultrasonographic measured optic nerve sheath diameter as an accurate and quick monitor for changes in intracranial pressure. J Neurosurg 123:743-747, 2015

20. March K: Intracranial pressure monitoring: why monitor? AACN Clin Issues 16:456-475, 2005

21. McKeating EG, Andrews PJ, Tocher JI, Menon DK: The intensive care of severe head injury: a survey of non-neurosurgical centres in the United Kingdom. Br J Neurosurg 12:7-14, 1998

22. Padayachy LC, Figaji AA, Bullock MR: Intracranial pressure monitoring for traumatic brain injury in the modern era. Childs Nerv Syst 26:441-452, 2010

23. Pople IK, Muhlbauer MS, Sanford RA, Kirk E: Results and complications of intracranial pressure monitoring in 303 children. Pediatr Neurosurg 23:64-67, 1995

24. Raboel PH, Bartek J Jr, Andresen M, Bellander BM, Romner B: Intracranial pressure monitoring: invasive versus non-invasive methods-a review. Crit Care Res Pract 2012:950393, 2012

25. Rajajee V, Vanaman M, Fletcher JJ, Jacobs TL: Optic nerve ultrasound for the detection of raised intracranial pressure. Neurocrit Care 15:506-515, 2011

26. Rebuck JA, Murry KR, Rhoney DH, Michael DB, Coplin WM: Infection related to intracranial pressure monitors in adults: analysis of risk factors and antibiotic prophylaxis. $\mathbf{J}$ Neurol Neurosurg Psychiatry 69:381-384, 2000

27. Rosenberg JB, Shiloh AL, Savel RH, Eisen LA: Non-invasive methods of estimating intracranial pressure. Neurocrit Care 15:599-608, 2011

28. Saladino A, White JB, Wijdicks EF, Lanzino G: Malplacement of ventricular catheters by neurosurgeons: a single institution experience. Neurocrit Care 10:248-252, 2009

29. Schimpf MM: Diagnosing increased intracranial pressure. J Trauma Nurs 19:160-167, 2012

30. Smith M: Monitoring intracranial pressure in traumatic brain injury. Anesth Analg 106:240-248, 2008

31. Steiner LA, Andrews PJ: Monitoring the injured brain: ICP and CBF. Br J Anaesth 97:26-38, 2006

32. Wakerley BR, Kusuma Y, Yeo LL, Liang S, Kumar K, Sharma AK, et al: Usefulness of transcranial Dopplerderived cerebral hemodynamic parameters in the noninvasive assessment of intracranial pressure. J Neuroimaging 25:111116,2015

33. Yuan Q, Wu X, Sun Y, Yu J, Li Z, Du Z, et al: Impact of intracranial pressure monitoring on mortality in patients with traumatic brain injury: a systematic review and metaanalysis. J Neurosurg 122:574-587, 2015 
34. Zhong J, Dujovny M, Park HK, Perez E, Perlin AR, Diaz FG: Advances in ICP monitoring techniques. Neurol Res 25:339-350, 2003

\section{Disclosures}

The authors report no conflict of interest concerning the materials or methods used in this study or the findings specified in this paper.

\section{Author Contributions}

Conception and design: Ganslandt. Acquisition of data:
Ganslandt, Mourtzoukos, Sommer, Rammensee. Analysis and interpretation of data: all authors. Drafting the article: Ganslandt, Stadlbauer, Sommer, Rammensee. Critically revising the article: Ganslandt, Stadlbauer, Rammensee. Reviewed submitted version of manuscript: Ganslandt. Approved the final version of the manuscript on behalf of all authors: Ganslandt. Study supervision: Ganslandt.

\section{Correspondence}

Oliver Ganslandt, Klinikum Stuttgart, Katharinenhospital, Kriegsbergstrasse 60, Stuttgart 70174, Germany. email: o.ganslandt@ klinikum-stuttgart.de. 